\title{
The admission of Asian patients to intensive therapy units and its implications for kidney donation: a preliminary report from Coventry, UK
}

\author{
Catherine Exley, Julius Sim, Norma G Reid, Lionel Booth, Simon Jackson, Nick West
}

\begin{abstract}
Objective - To determine the relative admission rates of Asian and non-Asian patients to intensive therapy units (ITUs) in Coventry and to explore the implications of these rates for the transplantation of organs to Asian people.
\end{abstract}

Design - Examination of 1991 census data and a retrospective review of ITU admissions books. Data were collected on ethnic background, presenting diagnosis, and clinical outcome for each admission.

Setting - The three ITUs in Coventry.

Patients - All admissions to the ITUs from 1990-93 inclusive.

Results - Asian patients were admitted to ITUs disproportionately to their numbers in the Coventry population. Members of the Asian community were less than half as likely to be admitted to an ITU $(p<0.001)$ and more likely to die while there $(p=0.007)$ than members of the nonAsian population in Coventry. The proportions of patients referred to the transplant unit and the rates of subsequent donation do not seem to differ significantly for Asian and non-Asian patients ( $p=0.26$ in both cases).

Conclusions - There are clear implications for the availability of cadaveric kidney transplantation to Asian patients, given that few kidneys from non-Asian donors are histocompatible with Asian recipients. Indeed, in Asians, promotion of living related donation may be more effective in countering the organ shortfall than efforts to increase consent to cadaveric transplantation. However, it may be valuable to investigate any patterns of morbidity or social or cultural factors that might explain the initial low admissions rates to ITUs for Asian patients.

(F Epidemiol Community Health 1996;50:447-450)

\section{School of Health an Social Sciences, Coventry University Priory Street, Coventry CVi 5FB. C Exley \\ J Sim \\ N G Reid}

Renal Unit, Walsgrave Hospital NHS Trust, Coventry

L Booth

S Jackson

$\mathrm{N}$ West

Correspondence to: Dr J Sim years, ${ }^{12}$ and has recently prompted fresh debate as to ways in which the accrual of organs might be increased, ${ }^{34}$ including the possibility of using organs from asystolic donors. $^{56}$

Although the present situation is serious for all members of the UK population in need of kidney transplants, it is particularly so for member of the Asian community, who characteristically have to wait considerably longer for a kidney than do those of European descent. Some reasons for this disparity are fairly well established. For example, there seems to be a higher incidence of diabetes and consequent diabetic nephropathy among the Asian community, ${ }^{7-9}$ including that in Coventry. ${ }^{10}$ Similarly, within the West Midlands the relative risk of end stage renal failure for Asian people compared with white people is of the order of 1.76. ${ }^{11}$ Recent figures specific to Coventry indicate that the annual incidence of end stage renal failure treated with replacement therapy is currently 589 per million population (pmp) for Asian people, the corresponding figure for the white population being $111 \mathrm{pmp} .^{12}$ This means that there is a greater demand for kidney transplants from the Asian population. There are also greater problems of histocompatibility and blood group matching. Thus, there are considerable differences in the prevalence of certain human leukocyte antigens (HLAs) between Asian and non-Asian populations, and whereas the $B$ blood group allele is most common in Asia, the $\mathrm{O}$ blood group allele tends to predominate in northern Europe. ${ }^{13}$ The result of these factors is that few organs donated by the white population in the UK are suitable for transplantation into Asian recipients.

A further factor underlying this situation is thought by some to be an unwillingness within the Asian community to consent to the removal of organs from deceased relatives. If this is so, it will indeed contribute to the relative shortage of kidneys for this section of the population. However, this rests upon the assumption that a similar proportion of Asian people become potential cadaveric donors as in the rest of the population. Clinical and professional experience within the research team suggested that this might not be the case, and the question arose as to whether a disproportionately small number of Asian people reach the situation in which they can be classed as a potential kidney donor. More specifically, it was hypothesised that Asian people do not find their way to intensive therapy units (ITUs) in 
the proportions one would expect and are therefore, by implication, less likely to become donors.

As part of a larger project investigating attitudes to organ donation among the Asian community in Coventry, a preliminary survey of the admissions into three ITUs was undertaken early in 1994, with the aim of establishing the number of Asian people who had been admitted into these units in the last four years. (The term Asian is here taken to mean all those whose racial origins are in the Indian subcontinent.) The study reported here was in the nature of a pilot study, to establish the initial plausibility of the proposed hypothesis before any further investigation within the wider project. No definitive test of the hypothesis was intended at this stage.

\section{Methods}

In order to establish baseline comparative data, the proportion of the Coventry population which is Asian was established from the 1991 census data. Although there may be under enumeration in census data, this is not thought to be selective to particular ethnic groups. ${ }^{14}$ Hence, these data should give a valid indication of the relative proportions of different ethnic populations.

Following this, and with the permission of the respective medical directors, the admission books for the three ITUs in the city were retrospectively reviewed from 1990-93 inclusive. The following points were noted for each year: total number of admissions; total number of Asian patients admitted, and the reason for admission; total number of deaths; and the total number of Asian patients who died. The fact that Coventry is relatively 'self sufficient' as regards medical services and the absence of another major city in the immediate vicinity, suggested that virtually all Asian patients in the city receiving ITU care during this period would have been admitted to one of the three units in the city.

The ITU admissions books were examined by the same member of the research team, who was also a member of hospital staff, and the ethnic origin of patients was determined by looking at both the religion which was recorded and the family name. All individuals whose religion was recorded as being Sikh, Hindu, or Muslim and who had a family name which was recognisable as Asian were counted in this sample. In the absence of a recorded religion, which was rare, the family name alone was used as the basis for classification. Full patient details, which would have allowed the identification of specific individuals, were not recorded in the admissions book and for the purposes of this pilot study there was no attempt to access the patients' medical records.

The resulting data were then cross referenced with the records of the Coventry Renal Transplant Unit, from which the total numbers of Asian and non-Asian referrals and of Asian and non-Asian donors were recorded for each year. Finally, the number of Asian patients currently waiting for a renal transplant in Coventry and the surrounding region was established
Table 1 Admissions to intensive therapy units in relation to year

\begin{tabular}{lcll}
\hline \multirow{5}{*}{ Admissions (\%) } \\
\cline { 2 - 4 } Year & \multicolumn{1}{l}{ Asian } & Non-Asian & Total \\
\hline 1990 & $52(4.3)$ & $1150(95.7)$ & 1202 \\
1991 & $46(3.8)$ & $1166(96.2)$ & 1212 \\
1992 & $48(3.8)$ & $1199(96.2)$ & 1247 \\
1993 & $54(3.8)$ & $1376(96.2)$ & 1430 \\
Total & $200(3.9)$ & $4891(96.1)$ & 5091
\end{tabular}

Table 2 Deaths in intensive therapy units in relation to year

\begin{tabular}{llll}
\hline \multirow{5}{*}{ Year } & \multicolumn{2}{l}{ Deaths (\%) } \\
\cline { 2 - 4 } & Asian & Non-Asian & Total \\
\hline 1990 & $5(3.4)$ & $144(96.6)$ & 149 \\
1991 & $9(7.3)$ & $114(92.7)$ & 123 \\
1992 & $9(5.9)$ & $144(94.1)$ & 153 \\
1993 & $12(7.4)$ & $150(92.6)$ & 162 \\
Total & $35(6)$ & $552(94)$ & 587
\end{tabular}

(this covers areas in the West Midlands Region, although there are obviously a few individuals from other areas who are referred). Additionally, the total number of patients who had been transplanted since 1990 and the proportion of these who were Asian were noted.

Statistical analysis of contingency tables was by means of calculating a $95 \%$ confidence interval (CI) for differences in percentages. The null hypothesis of no difference between these percentages was tested by means of the $\chi^{2}$ test; $p<0.05$ was taken as the critical value for statistical significance.

\section{Results}

The 1991 census data showed that of a total of 294387 people in the city of Coventry, 27623 (9.4\%) were Asian (7.3\% Indian, 1.3\% Pakistani, 0.4\% Bangladeshi, 0.4\% Asian other). This represents the largest ethnic minority population in Coventry.

Table 1 shows the ethnic origin of patients admitted to the ITUs over the four year period. The overall proportion of admissions from the Asian community was just under $4 \%$ (the stability of the yearly figures for these admissions perhaps bears some testimony to the reliability, if not the validity, of the data collection procedure used in the study). The difference between the percentage of Asian people admitted $(3.9 \%)$ and their representation in the city population $(9.4 \%)$ was $5.5 \%$ (95\% CI 4.9 , 6.0); this difference was statistically significant $\left(\chi^{2}=181.29, \mathrm{p}<0.001\right)$. The primary diagnoses of the admissions from the Asian population showed no noteworthy trends in the absence of comparative data and are not therefore reported here.

The numbers of deaths within the ITUs over this period are shown in table 2. Comparison of tables 1 and 2 shows that $17.5 \%$ of Asian patients admitted to the ITUs died there, compared with $11.3 \%$ of non-Asian patients; this difference of $6.2 \%(95 \%$ CI $0.8,11.5)$ was statistically significant $\left(\chi^{2}=7.27, \mathrm{p}=0.007\right)$.

Table 3 displays both the numbers of kidney donor referrals to the transplant unit from the three ITUs in Coventry for the period 
Table 3 Kidney donor referrals to the Coventry Renal Transplant Unit from Coventry intensive treatment units and subsequent donors, 1990-93

\begin{tabular}{|c|c|c|c|c|c|c|}
\hline \multirow[b]{2}{*}{ Year } & \multicolumn{3}{|c|}{ Referrals } & \multicolumn{3}{|c|}{ Donors } \\
\hline & Asian & Non-Asian & Total & Asian & Non-Asian & Total \\
\hline 1990 & 1 & 20 & 21 & 1 & 10 & 11 \\
\hline 1991 & 0 & 17 & 17 & 0 & 10 & 10 \\
\hline 1992 & 0 & 13 & 13 & 0 & 10 & 10 \\
\hline 1993 & 1 & 16 & 17 & 0 & 15 & 15 \\
\hline Total & 2 & 66 & 68 & 1 & 45 & 46 \\
\hline
\end{tabular}

Table 4 Patients currently awaiting renal transplantation in Coventry and the surrounding areas

\begin{tabular}{lrlr}
\hline \multirow{4}{*}{} & \multicolumn{2}{l}{ On waiting list (\%) } \\
\cline { 2 - 4 } & \multicolumn{1}{c}{ Asian } & Non-Asian & Total \\
\hline Coventry & $14(40)$ & $21(60)$ & 35 \\
Surrounds & $9(12)$ & $68(88)$ & 77 \\
Total & $23(21)$ & $89(79)$ & 112
\end{tabular}

concerned and the number of subsequent donors. Of the Asian patients who died, 5.7\% were referred to the transplant unit as potential kidney donors, whereas the corresponding figure for non-Asian patients who died was $12 \%$. This difference of $6.3 \%$ (95\% CI $-2.1,14.6)$ was not statistically significant $\left(\chi^{2}=1.25\right.$ $\mathrm{p}=0.26)$. Subsequent to referral, $2.9 \%$ of Asian patients who died became donors compared with $8.2 \%$ of corresponding non-Asian patients; this difference of $5.3 \%$ (95\% CI -0.7 , 11.3) was not statistically significant $\left(\chi^{2}=1.28\right.$, $\mathrm{p}=0.26)$.

Altogether 112 patients were registered with the transplant unit as waiting for renal transplants; table 4 gives details of their residence and ethnic origin. Over the period in question, 126 renal transplantation operations were performed in the transplant unit, of which $12(9.5 \%)$ were in Asian patients.

\section{Discussion}

The exploratory nature of this study and the relatively unsophisticated means of data collection employed in this preliminary phase do not permit a definitive test of the initial hypothesis. Nor is it possible to draw any firm inferences from the data on the reasons underlying the trends. Nonetheless, a number of interesting and significant points can be drawn from the above findings.

It is clear from the ITU admission data that people from the Asian community are admitted in much smaller proportions than one would expect given the size of this section of the population in Coventry. Allowing for possible shortcomings in both the census and the ITU data, it would be reasonable to expect twice this number of admissions over this period. Accordingly, the likelihood of a donor coming from the Asian community in Coventry appears to be low.

As table 2 shows, there are significantly more deaths in ITUs in Asian patients than in non-Asian patients. The relatively low admission rate to ITUs for Asian patients, combined with their relatively high death rate once admitted, suggests that there are factors which cause Asian people to be less likely to enter
ITUs and, if they do so, to enter at an advanced stage of illness and hence more likely to die than non-Asian patients. The reasons for this can only be speculated upon within the context of the present study, but a number of possibilities suggest themselves. The age structure of Asian populations in the UK at present is young in relation to that of the white populations. ${ }^{14}$ The fact that the mean age of those admitted to ITUs tends to be high could explain some of the disparity in admissions, though it would not account for the difference in death rates. It is also possible to hypothesise, in respect of the lower admission rates of Asian patients, that differences in lifestyle and behaviour or other social factors render them less likely than non-Asian people to be admitted to ITUs with life threatening conditions compatible with subsequent cadaveric kidney donation. This, however, would not necessarily explain the difference in death rates. Alternatively, the explanation for these findings may be connected with cultural, religious, or social factors influencing the uptake of ITU services. These factors might in some way inhibit or delay the entry of Asian people to ITUs and thereby explain both the low rate of initial admission and the high rate of subsequent death. The collection of comparative data on presenting diagnosis and other patient characteristics for Asian and non-Asian patients would shed some light on this issue.

It is worth noting that the relatively high death rate for Asian patients in ITUs may to some degree mitigate the effect on the number of donors of the low rate of admission among Asian people. A lower death rate would effectively mean that even fewer organs were available for cadaveric donation.

No significant differences were found between the referral rates of Asians and nonAsians to the transplant unit or between the subsequent donation rates of Asians and non-Asians. It seems, therefore, that religious or cultural impediments to organ donation per se had no discernible effect. Thus, the low rates of organ donation relative to the population seem to be related to the initial low admission rates. It seems, therefore, that the low rates of organ donation by Asian people may be rooted in cultural, religious, or social factors relating to the uptake of services of ITUs rather than by those relating to the donation of organs.

These preliminary results suggest that members of the Asian community are less than half as likely to be admitted to an ITU than non-Asian people. Thus, it may be that relatively few opportunities arise for Asian families to be approached for consent to cadaveric transplantation. Such findings. are obviously particularly significant when one further notes the fact that $21 \%$ of the patients waiting for renal transplants in Coventry and the surrounding areas and $40 \%$ of those waiting for renal transplants within the city of Coventry itself are from the Asian community. This evidence supports the contention that demand for organs from Asian patients outstrips the supply of organs most likely to be histocompatible, namely those available from 
the Asian community itself. Moreover, it is likely that the need of Asian patients for renal transplantation will steadily increase in the future. This is related to two factors: the direct relationship between age and the incidence of end stage renal failure and the fact that the difference in age structure between Asian and white populations in the UK is likely to diminish.

\section{Conclusion}

Clearly, more detailed and extensive studies than the present one must be conducted before any concrete recommendations can be made in respect of public policy on organ donation. However, it is reasonable to suggest that if the initial presentation of potential Asian kidney donors suggested by this study is confirmed, it may be that the promotion of living related donation is a more effective strategy to combat the shortage of suitable organs for Asian recipients than efforts to secure a higher rate of consent to cadaveric transplantation. This pilot study also raises more general but significant questions about service utilisation by ethnic minority groups in its finding of relatively low levels of admission to ITUs. These admission rates could be influenced by cultural, social, religious, and behavioural factors, as well as by morbidity levels and individual health status. It could be important to explore further the extent to which these factors explain the low admission rates for Asian people, and the extent to which policy interventions to change these factors are appropriate.

1 DHSS. Report of the Working Party on the Supply of Donor Organs for Transplantation. Conference of Medical Royal Colleges and their Faculties in the UK. London: Department of Health and Social Security, 1987.

2 Kennedy I. The donation and transplantation of kidneys: should the law be changed? In: Kennedy I. Treat me right: Essays in medical law and ethics. Oxford: Clarendon Press, 1988.

3 Kittur DS, Hogan M, Thukral VK, McGaw LJ, Alexander JW. Incentives for organ donation? Lancet 1991; 338: 1441-3.

4 Teo B. Is the adoption of more efficient strategies of organ procurement the answer to persistent organ shortage in transplantation? Bioethics 1992;6:113-29.

5 Gore SM, Hinds CJ, Rutherford AJ. Organ donation from intensive care units in England. BMF 1989;299:1193-7.

6 Wing AJ, Chang RWS. Non-heart beating donors as a source of kidneys. BMF 1994;308:549-50.

7 Mather HM, Keen H. The Southall diabetes survey: prevalence of known diabetes in Asians and Europeans. $B M \mathcal{F}$ 1985;291:1081-4.

8 Pazianas M, Eastwood JB, MacRae KD, Phillips ME. Racial origin and primary renal diagnosis in 771 patients with end-stage renal disease. Nephrol Dial Transplant 1991; 6:931-5.

9 Burden AC, McNally PG, Feehally J, Walls J. Increased incidence of end-stage renal failure secondary to diabetes mellitus in Asian ethnic groups in the United Kingdom. Diabetic Med 1992;9:641-5.

10 Simmons D, Williams DR, Powell M. Prevalence of diabetes in a predominantly Asian community: preliminary findin a predominantly Asian community: preliminary find-

11 Clark TJ, Richards NT, Adu D, Michael J. Increased prevalence of dialysis-dependent renal failure in ethnic minorities in the West Midlands. Nephrol Dial Transplant 1993;8:146-8.

12 Higgins RM, Edmunds ME, Dukes DC. End-stage renal failure in Indo-Asians. $Q \mathcal{F}$ Med 1995;88:523-4.

13 Polednak AP. Racial and ethnic differences in disease. New York: Oxford University Press, 1989.

14 Roderick PJ, Jones I, Raleigh VS, McGeown M, Mallick N. Population need for renal transplantation therapy in Thames Population need for renal transplantation therapy in
regions: ethnic dimension. $B M \mathcal{F} 1994 ; 309: 1111-14$ 